\title{
Role of district health authorities: a strategic future
}

The future has been pressed upon purchasers by the publication of the findings of the functions and manpower review, ${ }^{1}$ a report by the NHS Executive chiefly concerned to establish a strategic focus for the NHS. Its conclusions aim at securing the devolvement of many functions hitherto carried out at the centre or in the regional health authorities to district health authorities and family health services authorities. Yet in announcing the important new role for district health authorities in purchasing primary care the executive clearly identify this level of the new NHS as the key body through which strategy will be developed and discharged. The manpower review and the more recent executive letter ${ }^{2}$ set the groundwork for this development by announcing primary legislation to enable the merging of district health-authorities and family health services attributes and - in line with this thinking - heralding also the prospect of locally negotiated general practitioner (GP) contracts.

To date, the notion of purchasing has had mixed reviews. Its potential for addressing the challenge of developing a strategic function remains untested. The contracting of local services and service reviews have become embedded within an annual cycle of purchaser/ provider negotiations, yet the difficult business of changing the configuration of health services to address the healthcare needs of the 21 st century will require a strong managerial rather than administrative approach. In order to influence the quality of future health care district health authorities must learn to commission jointly with GP fundholders within an agreed and shared strategic focus; they must get alongside providers as far as possible and manage the market to ensure healthcare delivery which grasps the opportunities of health technologies, and, most difficult of all, they must gain public acceptance and confidence.

There is no doubt about the ambivalence of district health authorities towards having the greater part of their population covered by fundholders. Yet the relatively few district health authorities may have much to learn from the many more GP fundholders who have succeeded in making a difference to health services. These fundholders have made practical improvements to patient care both in primary care and by agreeing treatment protocols for secondary care referrals; they have cleared their waiting lists, focusing specifically on those waiting nine to 12 months or more, and they have managed to cap the demand for outpatient services (J Griffiths, NAHAT conference, Brighton, June 1994).

\section{Effects of GP fundholding}

A full evaluation of the effects of GP fundholding will be available in a forthcoming study from the Audit Commission. A key question, for strategic district health authorities, beyond the obvious ability of fundholders to shift funds from primary to secondary care, will be the appropriateness of the changes they make. How primary care practitioners experience health and illness among their practice population will undoubtedly mean that they place greater emphasis on morbidity, for example, in seeking better services for the care of musculoskeletal disorders, inflammatory joint disease, and minor mental health problems, than has hitherto been driving the priorities behind the current balance of investment in health care, which supports preferentially the acutely ill over those at risk of ill health or those with chronic illness. The prospect of shifts in health investment from the secondary to the primary care sector are to be welcomed, but only if the result is a better balance in relation to health needs and the capacity for health gain and not a refocusing of inefficiencies on the primary rather than the secondary care sector.

Ironically, GP fundholders, with their undoubted ability to negotiate with hospital based colleagues a better deal for patients, means that they are well placed to commission acute care. The prospect of GP fundholders as the professionals closest to the patient taking on the contracting process, particularly for elective surgery, with appropriate support from district health authorities and family health services authorities feels right. For chronic care, rehabilitation, long term support for elderly people and people with physical and mental handicaps community services may have a more important role working in partnership with district health authorities and social services to secure appropriate devolvement of budgets and a proper strategic focus.

The healthcare market is central to the development of purchasing. Comparing healthcare providers on the basis of price and quality has the potential to improve value for money. The difficulty is that trusts are not set up to be responsive to the strategic purchaser who is seeking the best outcomes from specialist providers and improved access for patients with common conditions from local providers. This will require greater specialisation of some services and decentralisation of others. At present, resources are locked up in the traditional district general hospital model, which often falls between the catchment population needed to secure the staffing and resources for offering high tech specialist care yet maintains excessive waiting lists for elective care because of its emergency workload. Resolving this conundrum will require changes in the organisation of health services to meet the needs of patients for short stays in specialist centres with full acute medical back up followed by transfer to more local settings for recovery and rehabilitation. Such an approach will require strong collaboration between purchasers and providers - clinicians and managers - in agreeing appropriate referral and discharge protocols to ensure desired outcomes.

\section{Public accountability}

Public accountability is being increasingly emphasised by the NHS executive clinical effectiveness initiative. ${ }^{3}$ The concepts underlying clinical effectiveness are readily accepted by clinicians and healthcare managers, though evidence of their practical application in purchasing healthcare is limited. An early effect is the increasing emphasis being given to the use of guidelines in clinical practice. ${ }^{4}$ Guidelines provide the framework to begin a dialogue between purchaser and provider on the quality 
of care in terms of the research evidence for effectiveness underlying clinical practice. This provides a better understanding of costs in terms of the care pathway, the skill mix needed to deliver care, and the treatments and drugs involved. Monitoring quality within this framework becomes a matter of identifying key "medical review criteria" ${ }^{5}$ or, more explicitly, those characteristics of the care process which most closely link the evidence with the outcome - for example, the proportion of patients receiving aspirin and $\beta$ blockers after myocardial infarction $^{6}$ and providing prophylactic antibiotic in caesarean section. ${ }^{7}$

More profoundly in terms of strategic change, the notion of clinical effectiveness will make explicit the choices for rationing healthcare. It is doubtful whether GPs will wish to take on the responsibility for limiting the availability of treatments even on the basis of poor proven effectiveness. Setting priorities for healthcare will need the approach of a strategic body and preferably one which is capable also of assessing the values placed by the local population on different options for resource allocation.

These reflections on the future and present challenges for purchasing health care make the strongest possible case for the pending merger of district health authorities and family health services authorities. Joint commissioning will overcome the boundaries which have hitherto dogged our ability to care for patients by condition or care group in the most appropriate care setting. There is no doubt that this development goes with the need for more resources to be invested in primary care and reorganisation of the secondary care sector. The long term future of district health authorities lies in the realm of healthcare strategy rather than contracting, which can be done only by those closest to the individual patient. The key to achieving the vision lies in convincing the public of the importance of establishing health services which focus purchasing invesment on improvements in healthcare outcomes.

Public Health Specialist,

ALISON FRATER

Hertfordshire Health Agency,

Welwyn Garden City,

Hertfordshire HL8 $6 \mathscr{F L}$

Managing the new NHS. Functions and responsibilities in the new NHS Leeds: NHS Executive, 1994.

NHS Management Executive. Improving clinical effectiveness Leeds: NHSME, 1994. (EL(94)74.)

NHS Management Executive. Improving the effectiveness of the NHS Leeds: NHSME, 1993. (EL(93)115.)

4 Grimshaw JM, Russell IT. Effects of clinical guidelines on medical practice. A systematic review of vigorous evaluations. Lancet 1993 342:1317-22.

5 Institute of Medicine. Guidelines for clinical practice from development to use Washington, DC: Academic Press, 1992.

6 ISIS-2 (Second International Study of Infarct Survival) Collaborative Group. Randomised trial of intravenous streptokinase, oral aspirin, both or neither among case of suspected acute myocardial infarction. Lancet 1988; ii:349-60.

Chalmers I, Enkin M, Keirse MINC, eds. Effective care in pregnancy and childbirth. Oxford: Oxford University Press, 1989. 